density of the Universe. Long-wavelength perturbations in a curvaton field could lead to significant modulation of smaller-scale power on the microwave-background sky, while satisfying the Grishchuk-Zel'dovich constraint. But this curvaton model necessarily leads to deviations from a Gaussian distribution of fluctuations, close to the $0.1 \%$ level $^{8}$.

Alternatively, Alexey Golovnev and colleagues have proposed ${ }^{9}$ a model of inflation driven by vector fields, which must be non-minimally coupled during inflation. The vector fields spontaneously break statistical isotropy, but a large number of vector fields could produce a distribution that is suffciently isotropic, or one could invoke a curvaton-type vector field which produces only the anisotropic part of the observed perturbations ${ }^{10,11}$.

Such elaborate models - dependent on additional fields and/or large-scale inhomogeneities - would be anathema to the pioneers of inflation in the 1980s, who dreamed of a simple inflation model that delivered a homogeneous and isotropic universe independent of any initial conditions. In the absence of quantitative data, theoretical cosmologists were naturally attracted to the simplest, most elegant models that invoked the smallest number of free parameters. But now, when cosmologists have more detailed data with which to probe the very early Universe, we may have to adjust our expectations to deal with the Universe as we find it.

Of course, the power asymmetry might prove to be an undiscovered systematic effect in the experiment, or due to some local astrophysical effect (the axis of maximum asymmetry is suspiciously close to the ecliptic axis, with an absence of power near the north ecliptic pole). So cosmologists eagerly await the launch this year of the European Space Agency's Planck satellite, which should provide an independent check of the temperature anisotropies on large angular scales while providing more detailed data on polarization and smaller angular scales. These, in turn, will enable improved tests of the Gaussian, or non-Gaussian, distribution of temperature anisotropies. We will want good reasons to accept a Universe that breaks our expectations of symmetry.
David Wands is at the Institute of Cosmology and Gravitation, University of Portsmouth,

Dennis Sciama Building, Burnaby Road,

Portsmouth PO1 3FX, UK.

e-mail:david.wands@port.ac.uk

\section{References}

1. Komatsu, E. et al. Astrophys. J. Suppl. Ser. (in the press); preprint at $<$ http://arxiv.org/abs/0803.0547> (2008.)

2. Bennett, C. L. et al. Astrophys. J. Suppl. Ser. 148, 1-27 (2003).

3. Land, K. \& Magueijo, J. Phys. Rev. Lett. 95, 071301 (2005).

4. Eriksen, H. K., Hansen, F. K., Banday, A. J., Gorski K. M. \& Lilje, P. B. Astrophys. J. 605, 14-20 (2004); erratum 609, 1198 (2004).

5. Hansen, F. K., Banday, A. J., Gorski, K. M., Eriksen, H. K. \& Lilje, P. B. Preprint at <http://arxiv.org/abs/0812.3795> (2008).

6. Erickcek, A. L., Kamionkowski, M. \& Carroll, S. M. Phys. Rev. D 78, 123520 (2008)

7. Grishchuk, L. P. \& Zel'dovich, Ya. B. Astron. Zh. 55, 209-215 (1978); English translation: Sov. Astron. 22, 125-129 (1978).

8. Lyth, D. H., Ungarelli, C. \& Wands, D. Phys. Rev. D 67, 023503 (2003)

9. Golovnev, A., Mukhanov, V. \& Vanchurin, V. J. Cosmol. Astropart. Phys. 0806, 009 (2008)

10. Yokoyama, S. \& Soda, J. J. Cosmol. Astropart. Phys. 0808, 005 (2008).

11. Dimopoulos, K., Lyth, D. H. \& Rodriguez, Y. Preprint at <http://arxiv.org/abs/0809.1055> (2008)

\title{
PHYSICS OF VIOLINS
}

\section{A little light music}

"A great violin is alive; its very shape embodies its maker's intentions, and its wood stores the history, or the soul, of its successive owners. I never play without feeling that I have released or, alas, violated spirits." So wrote the violinist Yehudi Menuhin, in his book Unfinished Journey. Would he say the same of the balsa-wood violin created by Chris Waltham (Am. J. Phys. 77, 30-35; 2009)?

Considered by many to be the 'perfect instrument', the violin of today shares the basic principles of its design with the first such instruments, which appeared in the mid-sixteenth century. The design was perfected over the next two centuries, most notably by the master luthiers of Cremona in Italy - the Stradivari, Guarneri and Amati families; little has changed since then.

Modern science, however, has revealed much about how the Cremonese masterpieces produce their superb sound. In the 1960s and 1970s, John C. Schelleng - who, after his retirement from Bell Laboratories as director of radio research, pursued his

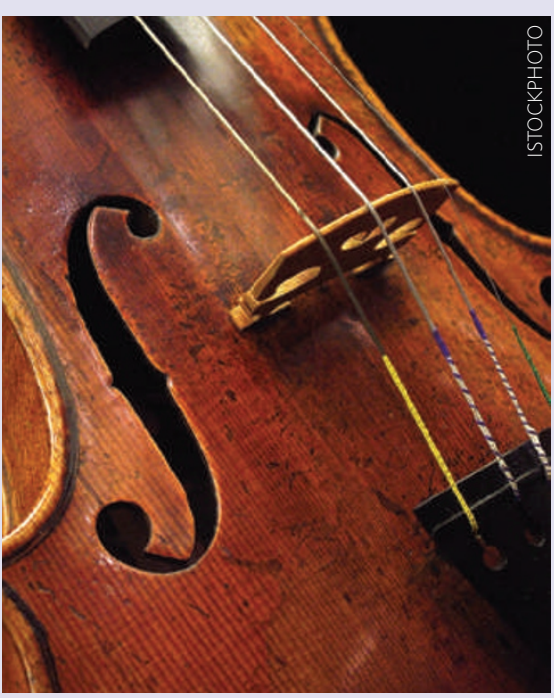

choices of spruce and maple in the construction of a violin. Still, Waltham's choice of balsa wood might seem unlikely, known as it is for the making of model aircraft rather than musical instruments.

It is actually not the first time that balsa wood has been used for violin-making, but those instruments also had innovative body shapes. Waltham, however, has stuck with the classic Stradivari design and is therefore able to verify Schelleng's scaling rules directly.

The key effect, when the top and back panels are replaced with balsa wood, comes from an altered mechanical impedance (a direct analogue to the a.c. electrical impedance). The mechanical impedance scales with the ratio between the density of the material and the speed of sound in the material, and its value is particularly low for balsa wood. This translates into a high volume of sound. The balsa violin is "distinctly loud", Waltham reports, and "a more appropriate instrument for a jig played in a noisy bar than for a soft lament."

ANDREAS TRABESINGER 